OPEN ACCESS

Edited by:

Yu Zhang,

VA Palo Alto Health Care System,

United States

Reviewed by:

Matteo Bologna,

Sapienza University of Rome, Italy

Maria A. Pastor,

University of Navarra, Spain

*Correspondence:

Patrice Péran

patrice.peran@inserm.fr

Specialty section:

This article was submitted to

Movement Disorders,

a section of the journal

Frontiers in Neurology

Received: 06 March 2020

Accepted: 25 June 2020

Published: 04 August 2020

Citation:

Arribarat G, De Barros A and Péran P (2020) Modern Brainstem MRI

Techniques for the Diagnosis of

Parkinson's Disease and

Parkinsonisms. Front. Neurol. 11:791.

doi: 10.3389/fneur.2020.00791

\section{Modern Brainstem MRI Techniques for the Diagnosis of Parkinson's Disease and Parkinsonisms}

\author{
Germain Arribarat ${ }^{1,2}$, Amaury De Barros ${ }^{1,3}$ and Patrice Péran ${ }^{1 *}$ \\ ${ }^{1}$ ToNIC, Toulouse Neurolmaging Center, Université de Toulouse, Inserm, UPS, Toulouse, France, ${ }^{2}$ Centre de Recherche \\ Cerveau et Cognition (CNRS, Cerco, UMR5549), UPS, Toulouse, France, ${ }^{3}$ Department of Anatomy, Toulouse Faculty of \\ Medicine, Toulouse, France
}

The brainstem is the earliest vulnerable structure in many neurodegenerative diseases like in Multiple System Atrophy (MSA) or Parkinson's disease (PD). Up-to-now, MRI studies have mainly focused on whole-brain data acquisition. Due to its spatial localization, size, and tissue characteristics, brainstem poses particular challenges for MRI. We provide a brief overview on recent advances in brainstem-related MRI markers in Parkinson's disease and Parkinsonism's. Several MRI techniques investigating brainstem, mainly the midbrain, showed to be able to discriminate PD patients from controls or to discriminate PD patients from atypical parkinsonism patients: iron-sensitive MRI, nigrosome imaging, neuromelanin-sensitive MRI, diffusion tensor imaging and advanced diffusion imaging. A standardized multimodal brainstem-dedicated MRI approach at high resolution able to quantify microstructural modification in brainstem nuclei would be a promising tool to detect early changes in parkinsonian syndromes.

Keywords: iron, neuromelanin (NM), multiple system atrophy (MSA), NODDI, diffusion kurtosis imaging (DKI), nigrosome

\section{INTRODUCTION}

In Parkinson's disease (PD), the first central neuropathological events occur in the brainstem and olfactory bulb. The dorsal motor nucleus of the vagal nerve in the medulla oblongata, which receives inputs from the neurenteric system, is classically considered to be the first nucleus involved before the ascending diffusion through other brainstem structures such as the serotoninergic raphe nuclei and noradrenergic coeruleus nuclei of the pons (1). Neurodegeneration then reaches the midbrain, and more particularly the dopaminergic substantia nigra pars compacta $(\mathrm{SNc})$ and cholinergic pedunculopontine nuclei (PPN). The SNc plays a pivotal role, with classic PD motor symptoms occurring when $30 \%$ or more of its dopaminergic neurons have disappeared (2).

Before the appearance of these motor symptoms, the spread of alpha-synuclein across the brainstem following the Braak stages is responsible for many non-motor symptoms, such as cardiac autonomic dysfunction, rapid eye movement behavior disorder, apathy, asthenia, depression and dysexecutive syndrome, arising from alteration of the different nuclei and white-matter bundles of the brainstem $(3,4)$. The ensuing SNc degeneration caused by disruption of serotoninergic, extranigral dopaminergic, cholinergic, and noradrenergic pathways leads to the limbic and cognitive network dysfunction that has been amply described in numerous molecular imaging studies $(5,6)$. Recent advances in structural MRI techniques, such as water molecular diffusion based techniques and neuromelanin (NM) or iron-sensitive sequences, are resulting in the ever more precise characterization of brainstem damage in $\mathrm{PD}$ and atypical parkinsonism, including 
a more precise comprehension of brainstem networks involved in non-motor symptoms. When extrapyramidal motor symptoms are present, the challenge is to differentiate true PD from atypical parkinsonian syndromes, the most challenging of these being progressive supranuclear paralysis (PSP), Parkinsonian or cerebellar variant of multiple system atrophy (MSAp, MSAc), and dementia with Lewy bodies (DLB). Red flags for these syndromes in otherwise normal routine MRI sequences for PD often take the form of anatomical structure atrophy, reflecting the massive neuronal loss and gliosis that generally characterize the more advanced stages (for a review of classic MRI signs for differential diagnosis, see (7).

As these red flags are already familiar to clinicians, the present review focuses on modern structural MRI techniques for PD diagnosis and the differential diagnosis of atypical parkinsonism, considering structures involved in motor networks, but also those involved in non-motor networks.

\section{NIGROSOME IMAGING}

Located in the midbrain, the susbtantia nigra (SN) is functionally and structurally divided into two parts: the $\mathrm{SNc}$ and the pars reticulata $(\mathrm{SNr})$. The rostroventral GABAergic $\mathrm{SNr}$ projects toward the thalamus, and the dopaminergic $\mathrm{SNc}$ toward the striatum. Calbindin immunohistochemistry has allowed a labeled nigral matrix and five unlabeled clusters or nigrosomes to be identified within the SNc. These are compartments of dopaminergic neurons where degeneration is particularly marked in PD (8-10). Five nigrosomes measuring up to several millimeters long have been described (11), the largest of which (Nigrosome-1) is found in the dorsal region of the SNc.

The structure of Nigrosome-1 has been successfully delineated on 7T MRI using high-resolution susceptibility weighted imaging (SWI) $(12,13)$. On SWI, this nigrosome has a hypersignal in the axial section, in either linear or comma form. It is bordered anteriorly, laterally and medially by a low-intensity signal, giving it a swallow tail appearance. The absence of this sign is viewed as a reliable diagnostic criterion for $\operatorname{PD}(12,14)$. Nigrosome-1 visualization at 7T has high diagnostic accuracy: sensitivity (100\%), specificity (87-100\%), positive predictive value (91-100\%), and negative predictive value (100\%) (15).

This structure has also been observed on SWI at 3T with reduced contrast $(14,16)$. The phase information, used here as a weighting mask, improves the visualization of the nigrosome's (16-18). This improvement stems from the difference in susceptibility between Nigrosome-1 and the surrounding nigral matrix in healthy individuals. As nigrosomes have a low iron concentration, they are visualized as a T2* hypersignal, contrasting with the nigral matrix. Recently, a meta-analysis reporting different nigrosome imaging techniques confirmed that visual assessment of dorsolateral nigral hyperintensity provides excellent diagnostic accuracy for PD vs. controls (19).

The two purported mechanisms behind the disappearance of the swallow tail sign are an increase in iron and a decrease in NM. A decrease in NM can cause a decrease in iron retention capacity, and therefore an increase in the amount of free iron. In both cases, the presence of iron induces paramagnetic properties of the signal (20).

\section{IRON-SENSITIVE IMAGING}

During the last decade, many works, using different ironsensitive MRI methods, confirmed the importance of nigral iron increase in PD patients compared to controls (21). Iron-sensitive MRI has several applications, especially in neurodegenerative disorders (22). Technically, iron content can be estimated in specific regions by measuring $\mathrm{T} 2$ and $\mathrm{T} 2 *$ relaxation rates, using either magnitude (R2*) (23-25) or phase (quantitative susceptibility mapping, QSM) imaging (26). QSM method demonstrated to be the most sensitive quantitative technique for detecting a significant increase of iron for PD (27). QSM is able to detect nigral iron increase even in prodromal stage of PD such as idiopathic rapid eye movement sleep behavior disorder (28). It is important to note that iron-content in the substantia nigra do not differ between $\mathrm{PD}$ and multiple system atrophy (MSA) patients (i.e., patient with atypical parkinsonism), and between MSA variants (29). Accordingly, studies have shown that QSM more closely reflects levodopa dosage and disease severity $(30,31)$.

Concerning the differential diagnosis between PD and atypical parkinsonism's, QSM techniques allow for more sensitive measures in the nigral and extranigral regions. Mazzucchi et al. (32) found that the greatest diagnostic accuracy for PSP was for increased $\chi$ values in the RN, subthalamic nucleus (STN), and medial part of the SN, whereas for MSA, it was a significantly higher level of iron deposition in the putamen, reflecting the different patterns of pathological involvement that characterize these diseases (32).

\section{NEUROMELANIN-SENSITIVE IMAGING}

NM is a dark pigment composed of melanin, proteins, lipids and metal ions $(33,34)$. NM-containing neurons are particularly concentrated in the SNc and locus coeruleus (LC). This pigment is found in both the nigral matrix and the nigrosomes. The MR signal in NM-containing neurons is paramagnetic and therefore allows easy MR imaging where it is sensitive to this type of signal (35-37). T1-weighted NM-sensitive MRI (NMMRI) produces hyperintense signals in regions containing NM. Indeed, the primary mechanism underlying contrast in NM-MRI appears to be the T1 reduction associated with melanin-iron complexes (38).

Studies have confirmed that patients with PD have a significantly reduced NM signal in the SN and LC (20, 37, 37, 39-41). These signal changes have been found to be correlated with the absorption values of the nigrostriatal dopamine transporter (42). In this same study, volumetric analysis of the NM-related signal also revealed a significant degree of atrophy. Measurement of NM-sensitive images has high diagnostic accuracy for PD. Several teams have recently evaluated a multimodal methodological approach, combining diffusion and NM-sensitive MRI. Pyatigorskaya et al. (43) concluded that 


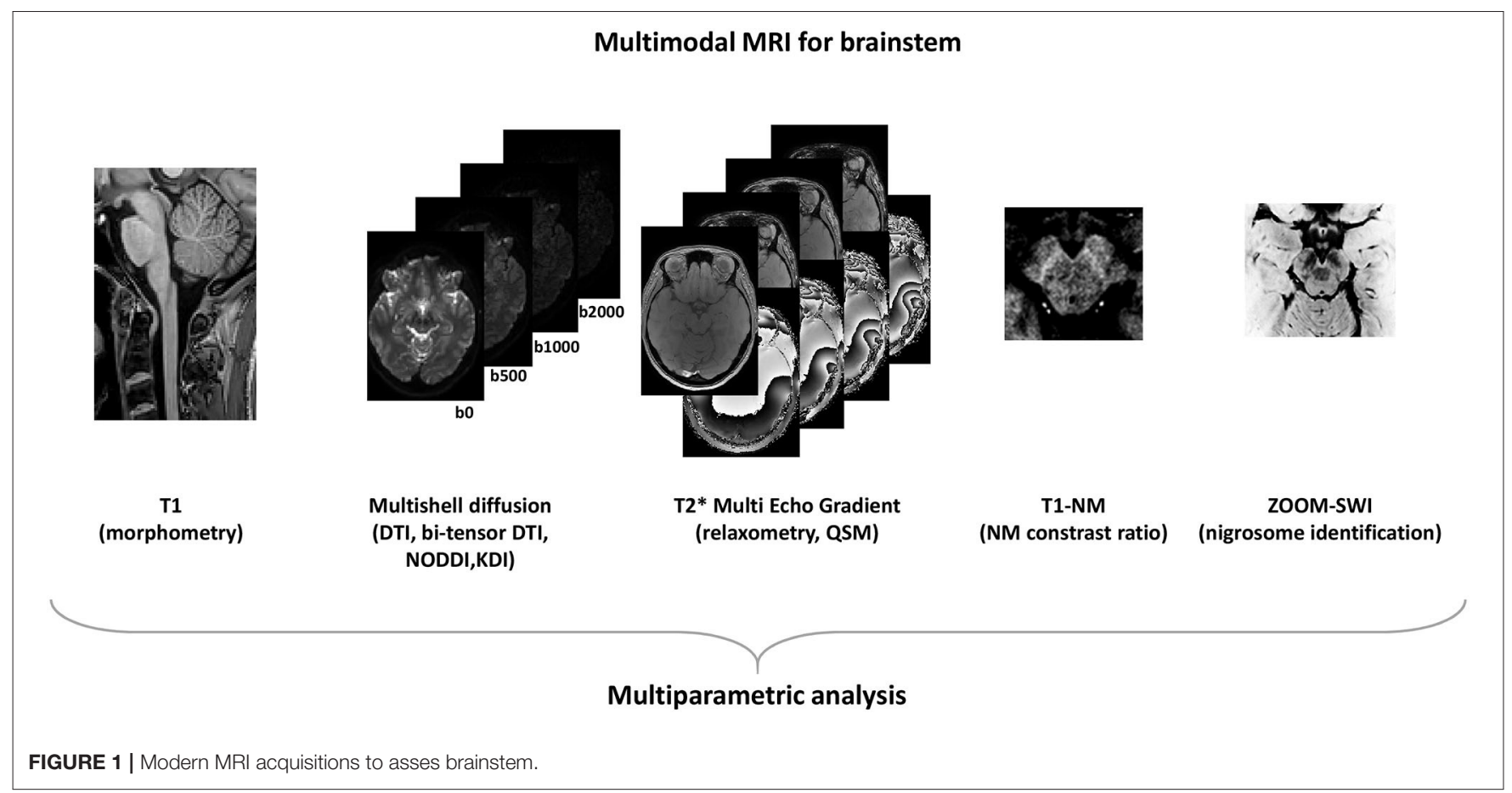

volume delineation by the NM signal, combined with fractional anisotropy, has excellent diagnostic accuracy for PD. More specifically, NM signal intensities can potentially be located in the SNc (43). Another study found a lower NM signal in PD and MSAp groups than in an PSP group, with lower intensities in the LC in patients with PD. Sensitivity and specificity were 60 and $90 \%$ for PD vs. MSAp, $63-88 \%$ and $77-92 \%$ for PD vs. PSP, and 80 and $85 \%$ for MSAp vs. PSP (44).

\section{DIFFUSION WEIGHTED IMAGING}

\section{Diffusion Tensor Imaging (DTI)}

There are conflicting findings concerning the usefulness of SN DTI metrics in PD diagnosis, according to recent metaanalyses (45-47) and recent voxelwise analysis-based studies concerning the whole SN (48-50). Fractional Anisotropy (FA) in the posterior part seems to be the most discriminant feature $(51,52)$. With the ADC for the whole SN, Zhong et al. (53) found accuracy of 0.72 , which is not sufficient in routine diagnosis. Interestingly, DTI metrics have also been used in a longitudinal approach, providing precious information on structural changes in the SN over the years (53-56).

In a longitudinal study applying a voxelwise approach, Pozorski et al. (50) found that patients with PD and controls differed on all DTI metrics in different brainstem regions (midbrain and pontine tegmentum, pontine crossing tract, periaqueductal gray matter). Furthermore, Mean diffusivity (MD) in the brainstem were negatively associated with disease duration. Pyatigorskaya et al. (57) correlated DTI metrics in the medulla oblongata with cardiac and respiratory variability in patients with PD compared with controls, reflecting their autonomic dysfunction (Stage 1) (57). Prange et al. (58) correlated apathy and depression with DTI measures and serotoninergic molecular imaging in the limbic system, and with the mode of anisotropy (sensitive to the orientation of crossing fibers) in the caudal midbrain, or more specifically the serotoninergic raphe nuclei (Stage 2) (58). Rapid eye movement behavior disorder has also been linked to DTI changes in the pons, SN and LC (59-61). Freezing of gait has been correlated with an FA decrease and MD increase in the PPN (62).

Diffusivity in the striatum, brainstem and cerebellum has been extensively studied $(7,63)$, but with rather mediocre results for the differential diagnosis of PD. FA or MD in the brainstem can be included in a multimodal approach with relatively good accuracy $(49,64)$. For example, the area under the curve (AUC) was above 0.95 for the diagnosis of PD vs. MSA in Péran et al. (49). Combining FA and MD in multiple ROIs including brainstem structures, $\mathrm{Du}$ et al. (51) were able to discriminate parkinsonian syndromes. With R2*, the AUC rose to 0.98-0.99. Pyatigorskaya et al. (43) recently found substantial differences in FA between patients with PSP and patients with PD or controls in the SN, LC, midbrain tegmentum, and pons. The highest AUC for PD vs. PSP was in the LC (0.94). In the same vein, Talai et al. (65) found AUCs of 0.95 and 0.97 based on DTI metrics in the midbrain for PD vs. PSP. DTI abnormalities in the midbrain of patients with PSP reflect microstructural changes that precede the macrostructural changes revealed by midbrain atrophy (65).

\section{Advanced Diffusion Approaches}

Despite unique insights yielded by DTI metrics acquired in a single shell, microstructural changes are considered non-specific.

Recently, bi-tensor model have been used on diffusion imaging is to differentiate free water (FW) (cerebrospinal fluid or extracellular vasogenic edema) from other compartments, 
and either eliminate it from DTI measures or directly map it. Although it was initially developed with a single shell (66), double-shell acquisition could offer greater accuracy and stability (67). The most striking feature is the FW increase in the posterior part of the SN in early PD (68), MSA and PSP (69), as confirmed by (70). Findings of longitudinal FW changes over 4 years in the posterior SN of patients with early PD (71), followed by an FW increase over 3 years in the anterior SN of patients with advanced PD (72), emphasize the usefulness of FW imaging for PD diagnosis and follow-up. This posterioranterior gradient of extracellular space expansion could reflect the neuroinflammation and cell loss revealed by histopathology. Increased FW in the posterior SN has been correlated with striatal dopaminergic denervation and reflects both motor and cognitive deficits (73). Planetta et al. (74) found an FW increase in the SN, as well as in the STN, red nucleus, PPN, cerebellum and basal ganglia, in patients with PSP and MSA, compared with patients with PD and controls. FW in the PPN and STN was discriminant for the diagnosis of MSA versus PSP (AUC $=0.97)(74)$.

Estimating kurtosis provides a better means of assessing diffusion heterogeneity in tissues. Diffusion kurtosis imaging (DKI) requires diffusion acquisition in two shells (often $b=1,000$ and 2,000 s/mm2) (75). Elevated mean kurtosis in the $\mathrm{SN}$ has been found to be more sensitive than FA reduction in patients with $\mathrm{PD}$ versus controls (AUC > 0.95) and is correlated with motor scores (76).

White-matter tissue has been simplified in a model with intraaxonal, extra-axonal, and cerebrospinal fluid compartments. Neurite orientation dispersion and density imaging (NODDI) takes all three compartments into account. Three new metrics can be extracted from NODDI: isotropic volume fraction (Viso), reflecting extracellular space like FW; intracellular volume fraction (Vic); and orientation dispersion index (ODI), reflecting neurite integrity. An ODI increase in white matter reflects axonal loss or disorganization, while ODI and Vic decreases in graymatter structures reflect dendritic thinning and density (77). Kamagata et al. (78) found that Vic in the contralateral SNc is useful for diagnosing patients with PD vs. controls $(\mathrm{AUC}=0.91)$ and correlates with disease severity (78). NODDI was recently compared with FW for diagnosing PD, PSP and MSAp. For $\mathrm{PD}$ and MSAp, Viso in the posterior SN was increased (FW accumulation). In PSP, all three NODDI metrics were disturbed in the whole SN, STN, RN and PPN. Interestingly, the authors considered the NODDI metrics to be inferior to FW for PD vs. MSAp/PSP diagnosis, and found that FW based on a single shell was just as accurate and actually faster than multishell FW (79).

Restriction spectrum imaging (RSI) $(80,81)$ is a new diffusion model based on high angular resolution diffusion imaging (HARDI), acquired in multiple directions at different high $\mathrm{b}$ values. RSI highlights hindered and restricted diffusion in extracellular and intracellular compartments. With the development of several new metrics, RSI can now provide information about neurite density and orientation. Hope et al. (81) were the first to compare RSI with DTI in patients with PD.
They defined a cellularity index and a neurite density index. In an ROI comprising the whole brainstem, they found significant differences in the cellularity index between patients with PD and controls (AUC $=0.69$ ). RSI is a promising new, but timeconsuming, method for vulnerable patients. Further research with more precise anatomical ROIs is needed.

DWI offers the possibility of visualizing white-matter tracts with a multitude of tractography techniques. HARDI-based tractography allows complex fiber tracts such as the nigrostriatal pathways (NSPs) to be reconstructed $(82,83)$. An FA decrease and an $\mathrm{AD} / \mathrm{RD}$ increase have been found in the NSPs of patients with PD using deterministic tractography (84). Based on NODDI, Andica et al. (85) found that the contralateral distal (relative to the striatum) Vic of NSPs was significantly decreased in patients with $\mathrm{PD}$ compared with controls, reflecting the dying back of dopaminergic neurons (85). From our point of view, the deterministic tractography of NSPs described in these studies does not render the exact structural connectivity between the striatum, pallidum and SN revealed by a probabilistic approach (82) or by susceptibility imaging (86). The tract-based approach to $\mathrm{PD}$ diagnosis is promising but needs further clarification of mesencephalon/diencephalon structural connectivity.

The dentatorubrothalamic tract (DRTT) connects the cerebellum with the thalamus via the superior cerebellar peduncle and tegmental midbrain. An FA decrease and an MD increase have been found in the DRTT of patients with PSP, compared with controls and patients with PD or MSAp $(64,87)$. In patients with MSAc, an FA decrease and an MD increase in the pontine crossing tract and middle cerebellar peduncles have been found before the appearance of the hot cross bun sign $(88,89)$. Using diffusion kurtosis imaging, Ito et al. $(90,91)$ described a ratio of midbrain tegmentum diffusion to pontine crossing tract diffusion to distinguish between patients with PSP, MSA or PD and controls $(90,91)$. Interestingly, Juttukonda et al. (92) also found an FA decrease in the pontine crossing tract in patients with essential tremor vs. PD (92).

\section{CONCLUSION}

An important consideration is that MRI quantitative markers with good performances for diagnosis are not necessarily the best suited to monitor disease progression. More efforts need to be done in order to increase reliably and sensitivity of progression MRI markers of PD (93). A standardized multimodal brainstemdedicated MRI approach at high spatial resolution (Figure 1) able to quantify microstructural modification in brainstem nuclei would be a promising tool to detect early changes in PD and parkinsonism and to follow disease progression.

\section{AUTHOR CONTRIBUTIONS}

$A D, G A$, and PP contributed conception and design of the review. $\mathrm{AD}$ and GA wrote the first draft of the manuscript. PP reviewed the first draft. All authors contributed to manuscript revision, read, and approved the submitted version. 


\section{REFERENCES}

1. Braak H, Ghebremedhin E, Rüb U, Bratzke H, Tredici K. Stages in the development of \{Parkinson\}'s disease-related pathology. Cell Tissue Res. (2004) 318:121-34. doi: 10.1007/s00441-004-0956-9

2. Cheng $\mathrm{H}-\mathrm{C}$, Ulane $\mathrm{CM}$, Burke RE. Clinical progression in Parkinson disease and the neurobiology of axons. Ann Neurol. (2010) 67:715-25. doi: 10.1002/ana.21995

3. Grinberg LT, Rueb U, Alho AT, di L, Heinsen H. Brainstem pathology and non-motor symptoms in PD. J Neurol Sci. (2010) 289:81-8. doi: 10.1016/j.jns.2009.08.021

4. Seidel K, Mahlke J, Siswanto S, Krüger R, Heinsen H, Auburger G, et al. The brainstem pathologies of parkinson's disease and dementia with lewy bodies. Brain Pathol. (2015) 25:121-35. doi: 10.1111/bpa.12168

5. Strafella AP, Bohnen NI, Perlmutter JS, Eidelberg D, Pavese N, Van Eimeren T, et al. Molecular imaging to track Parkinson's disease and atypical parkinsonisms: new imaging frontiers. Mov Disord. (2017) 32:181-92. doi: $10.1002 / \mathrm{mds} .26907$

6. Strafella AP, Bohnen NI, Pavese N, Vaillancourt DE, Eimeren T, Politis M, et al. Imaging markers of progression in parkinson's disease. Mov Disord Clin Pract. (2018) 5:586-96. doi: 10.1002/mdc3.12673

7. Heim B, Krismer F, De Marzi R, Seppi K. Magnetic resonance imaging for the diagnosis of Parkinson's disease. J Neural Transm. (2017) 124:915-64. doi: 10.1007/s00702-017-1717-8

8. Hirsch E, Graybiel AM, Agid YA. Melanized dopaminergic neurons are differentially susceptible to degeneration in Parkinson's disease. Nature. (1988) 334:345-8. doi: 10.1038/334345a0

9. Damier P, Hirsch EC, Agid Y, Graybiel AM. The substantia nigra of the human brain. I. Nigrosomes and the nigral matrix, a compartmental organization based on calbindin $\mathrm{D}(28 \mathrm{~K})$ immunohistochemistry. Brain. (1999) 122(Pt 8):1421-36. doi: 10.1093/brain/122.8.1421

10. Damier P, Hirsch EC, Agid Y, Graybiel AM.. The substantia nigra of the human brain. II. Patterns of loss of dopamine-containing neurons in Parkinson's disease. Brain. (1999) 122(Pt 8):1437-48. doi: 10.1093/brain/122.8.1437

11. Schwarz ST, Mougin O, Xing Y, Blazejewska A, Bajaj N, Auer DP, et al. Parkinson's disease related signal change in the nigrosomes 1-5 and the substantia nigra using T2* weighted 7T MRI. NeuroImage Clin. (2018) 19:683-9. doi: 10.1016/j.nicl.2018.05.027

12. Blazejewska AI, Schwarz ST, Pitiot A, Stephenson MC, Lowe J, Bajaj N, et al. Visualization of nigrosome 1 and its loss in PD: Pathoanatomical correlation and in vivo $7 \mathrm{~T}$ MRI. Neurology. (2013) 81:534-40. doi: 10.1212/WNL.0b013e31829e6fd2

13. Lehéricy S, Bardinet E, Poupon C, Vidailhet M, François C. 7 tesla magnetic resonance imaging: a closer look at substantia nigra anatomy in Parkinson's disease. Mov Disord. (2014) 29:1574-81. doi: 10.1002/mds.26043

14. Schwarz ST, Afzal M, Morgan PS, Bajaj N, Gowland PA, Auer DP. The "swallow tail" appearance of the healthy nigrosome - a new accurate test of Parkinson's disease: a case-control and retrospective cross-sectional MRI study at 3T. PLoS ONE. (2014) 9:93814. doi: 10.1371/journal.pone.0093814

15. Cosottini M, Frosini D, Pesaresi I, Costagli M, Biagi L, Ceravolo R, et al. MR imaging of the substantia nigra at $7 \mathrm{~T}$ enables diagnosis of parkinson disease. Radiology. (2014) 271:831-8. doi: 10.1148/radiol.14131448

16. Gao P, Zhou PY, Li G, Zhang GB, Wang PQ, Liu JZ, et al. Visualization of nigrosomes-1 in 3T MR susceptibility weighted imaging and its absence in diagnosing Parkinson's disease. Eur Rev Med Pharmacol Sci. (2015) 19:4603-9.

17. Nam Y, Gho SM, Kim DH, Kim EY, Lee J. Imaging of nigrosome 1 in substantia nigra at 3T using multiecho susceptibility map-weighted imaging (SMWI). J Magn Reson Imaging. (2017) 46:528-36. doi: 10.1002/jmri.25553

18. Jo $\mathrm{M}, \mathrm{Oh} \mathrm{S}$. A preliminary attempt to visualize nigrosome 1 in the subtantia nigra for parkinson's disease at 3T: an efficient susceptibility map-weighted imaging (SMWI) with quantitative susceptibility mapping using deep neural network (QSMnet). Med Phys. (2019) 47, 604-613. doi: 10.1002/mp. 13999

19. Mahlknecht P, Krismer F, Poewe W, Seppi K. Meta-analysis of dorsolateral nigral hyperintensity on magnetic resonance imaging as a marker for Parkinson's disease. Mov Disord. (2017) 32:619-23. doi: 10.1002/mds.26932
20. Takahashi H, Watanabe Y, Tanaka H, Mihara M, Mochizuki H, Liu T, et al. Quantifying changes in nigrosomes using quantitative susceptibility mapping and neuromelanin imaging for the diagnosis of early-stage Parkinson's disease. Br J Radiol. (2018) 91:20180037. doi: 10.1259/bjr.20180037

21. Guan X, Xu X, Zhang M. Region-specific iron measured by MRI as a biomarker for parkinson's disease. Neurosci Bull. (2017) 33:561-7. doi: $10.1007 / \mathrm{s} 12264-017-0138-\mathrm{x}$

22. De Barros A, Arribarat G, Combis J, Chaynes P, Péran P. Matching ex vivo MRI with Iron histology: pearls and pitfalls. Front Neuroanat. (2019) 13:68. doi: 10.3389/fnana.2019.00068

23. Péran P, Hagberg G, Luccichenti G, Cherubini A, Brainovich V, Celsis P, et al. Voxel-based analysis of R2* maps in the healthy human brain. J Magn Reson Imaging. (2007) 26:1413-20. doi: 10.1002/jmri.21204

24. Péran P, Cherubini A, Luccichenti G, Hagberg G, Démonet J-F, Rascol O, et al. Volume and iron content in basal ganglia and thalamus. Hum Brain Mapp. (2009) 30:2667-75. doi: 10.1002/hbm.20698

25. Cherubini A, Péran P, Caltagirone C, Sabatini U, Spalletta G. Aging of subcortical nuclei: microstructural, mineralization and atrophy modifications measured in vivo using MRI. Neuroimage. (2009) 48:29-36. doi: 10.1016/j.neuroimage.2009.06.035

26. De Rochefort L, Liu T, Kressler B, Liu J, Spincemaille P, Lebon V, et al. Quantitative susceptibility map reconstruction from MR phase data using bayesian regularization: validation and application to brain imaging. Magn Reson Med. (2010) 63:194-206. doi: 10.1002/mrm.22187

27. Barbosa JHO, Santos AC, Tumas V, Liu M, Zheng W, Haacke EM, et al. Quantifying brain iron deposition in patients with Parkinson's disease using quantitative susceptibility mapping, R2 and R2*. Magn Reson Imaging. (2015) 33:559-65. doi: 10.1016/j.mri.2015.02.021

28. Sun J, Lai Z, Ma J, Gao L, Chen M, Chen J, et al. Quantitative evaluation of iron content in idiopathic rapid eye movement sleep behavior disorder. Mov Disord. (2019) 35:478-85. doi: 10.1002/mds.27929

29. Barbagallo G, Sierra-Peña M, Nemmi F, Traon AP, Meissner L, Rascol WG, et al. Multimodal MRI assessment of nigro-striatal pathway in multiple system atrophy and Parkinson disease. Mov Disord. (2016) 31:325-34. doi: $10.1002 / \mathrm{mds} .26471$

30. Langkammer C, Pirpamer L, Seiler S, Deistung A, Schweser F, Franthal S, et al. Quantitative susceptibility mapping in parkinson's disease. PLoS ONE. (2016) 11:e0162460. doi: 10.1371/journal.pone.0162460

31. Ropele S, Langkammer C. Iron quantification with susceptibility. NMR Biomed. (2017) 30:e3534. doi: 10.1002/nbm.3534

32. Mazzucchi S, Frosini D, Costagli M, Del Prete E, Donatelli G, Cecchi P, et al. Quantitative susceptibility mapping in atypical Parkinsonisms. NeuroImage Clin. (2019) 24:101999. doi: 10.1016/j.nicl.2019.101999

33. Zecca L, Shima T, Stroppolo A, Goj C, Battiston GA, Gerbasi R, et al. Interaction of neuromelanin and iron in substantia nigra and other areas of human brain. Neuroscience. (1996) 73:407-15. doi: 10.1016/0306-4522(96)00047-4

34. Zecca L, Bellei C, Costi P, Albertini A, Monzani E, Casella L, et al. New melanic pigments in the human brain that accumulate in aging and block environmental toxic metals. Proc Natl Acad Sci USA. (2008) 105:17567-72. doi: 10.1073/pnas.0808768105

35. Sasaki M, Shibata E, Tohyama K, Takahashi J, Otsuka K, Tsuchiya K, et al. Neuromelanin magnetic resonance imaging of locus ceruleus and substantia nigra in Parkinson's disease. Neuroreport. (2006) 17:1215-8. doi: 10.1097/01.wnr.0000227984.84927.a7

36. Sasaki M, Shibata E, Kudo K, Tohyama K. Neuromelanin-\{Sensitive $\{$ MRI . Clin Neuroradiol. (2008) 18:147-53. doi: 10.1007/s00062-008-8018-4

37. Sulzer D, Cassidy C, Horga G, Kang UJ, Fahn S, Casella L, et al. Neuromelanin detection by magnetic resonance imaging (MRI) and its promise as a biomarker for Parkinson's disease. NPJ Park Dis. (2018) 4:11. doi: 10.1038/s41531-018-0047-3

38. Trujillo P, Summers PE, Ferrari E, Zucca FA, Sturini M, Mainardi LT, et al. Contrast mechanisms associated with neuromelanin-MRI. Magn Reson Med. (2017) 78:1790-800. doi: 10.1002/mrm.26584

39. Ogisu K, Kudo K, Sasaki M, Sakushima K, Yabe I, Sasaki H, et al. 3D neuromelanin-sensitive magnetic resonance imaging with semiautomated volume measurement of the substantia nigra pars compacta 
for diagnosis of Parkinson's disease. Neuroradiology. (2013) 55:719-24. doi: 10.1007/s00234-013-1171-8

40. Castellanos G, Fernández-Seara MA, Lorenzo-Betancor O, Ortega-Cubero $\mathrm{S}$, Puigvert $\mathrm{M}$, Uranga $\mathrm{J}$, et al. Automated neuromelanin imaging as a diagnostic biomarker for parkinson's disease. Mov Disord. (2015) 30:945-52. doi: 10.1002/mds.26201

41. Pavese N, Tai YF. Nigrosome imaging and neuromelanin sensitive MRI in diagnostic evaluation of parkinsonism. Mov Disord Clin Pract. (2018) 5:131-40. doi: $10.1002 / \mathrm{mdc} 3.12590$

42. Kuya K, Ogawa T, Shinohara Y, Ishibashi M, Fujii S, Mukuda N, et al. Evaluation of Parkinson's disease by neuromelanin-sensitive magnetic resonance imaging and 123I-FP-CIT SPECT. Acta Radiol. (2018) 59:593-8. doi: $10.1177 / 0284185117722812$

43. Pyatigorskaya N, Magnin B, Mongin M, Yahia-Cherif L, Valabregue R, Arnaldi D, et al. Comparative study of MRI biomarkers in the substantia nigra to discriminate idiopathic Parkinson disease. Am J Neuroradiol. (2018) 39:14607. doi: 10.3174/ajnr.A5702

44. Ohtsuka C, Sasaki M, Konno K, Kato K, Takahashi J, Yamashita F, et al. Differentiation of early-stage parkinsonisms using neuromelaninsensitive magnetic resonance imaging. Park Relat Disord. (2014) 20:755-60. doi: 10.1016/j.parkreldis.2014.04.005

45. Atkinson-Clement C, Pinto S, Eusebio A, Coulon O. Diffusion tensor imaging in Parkinson's disease: review and meta-analysis. NeuroImage Clin. (2017) 16:98-110. doi: 10.1016/j.nicl.2017.07.011

46. Hirata FCC, Sato JR, Vieira G, Lucato LT, Leite CC, Bor-Seng-Shu E, et al. Substantia nigra fractional anisotropy is not a diagnostic biomarker of Parkinson's disease: a diagnostic performance study and meta-analysis. Eur Radiol. (2017) 27:2640-8. doi: 10.1007/s00330-016-4611-0

47. Deng X-Y, Wang L, Yang T-T, Li R, Yu G. A meta-analysis of diffusion tensor imaging of substantia nigra in patients with Parkinson's disease. Sci Rep. (2018) 8:2941. doi: 10.1038/s41598-018-20076-y

48. Haehner A, Schöpf V, Loureiro A, Linn J, Reichmann H, Hummel T, et al. Substantia nigra fractional anisotropy changes confirm the PD at-risk status of patients with idiopathic smell loss. Park Relat Disord. (2018) 50:113-6. doi: 10.1016/j.parkreldis.2018.02.026

49. Péran P, Barbagallo G, Nemmi F, Sierra M, Galitzky M, Traon AP-L, et al. MRI supervised and unsupervised classification of Parkinson's disease and multiple system atrophy. Mov Disord. (2018) 33:600-8. doi: 10.1002/mds.27307

50. Pozorski V, Oh JM, Adluru N, Merluzzi AP, Theisen F, Okonkwo O, et al. Longitudinal white matter microstructural change in Parkinson's disease. Hum Brain Mapp. (2018) 39:4150-61. doi: 10.1002/hbm.24239

51. Du G, Lewis MM, Kanekar S, Sterling NW, He L, Kong L, et al. Combined diffusion tensor imaging and apparent transverse relaxation rate differentiate Parkinson disease and atypical parkinsonism. Am J Neuroradiol. (2017) 38:966-72. doi: 10.3174/ajnr.A5136

52. Knossalla F, Kohl Z, Winkler J, Schwab S, Schenk T, Engelhorn T, et al. Highresolution diffusion tensor-imaging indicates asymmetric microstructural disorganization within substantia nigra in early Parkinson's disease. J Clin Neurosci. (2018) 50:199-202. doi: 10.1016/j.jocn.2018.01.023

53. Zhong Z, Merkitch D, Karaman MM, Zhang J, Sui Y, Goldman JG, et al. High-spatial-resolution diffusion MRI in parkinson disease: lateral asymmetry of the substantia nigra. Radiology. (2019) 291:149-57. doi: 10.1148/radiol.2019181042

54. Zhang Y, Wu IW, Tosun D, Foster E, Schuff N. Progression of regional microstructural degeneration in Parkinson's disease: a multicenter diffusion tensor imaging study. PLoS ONE. (2016) 11:e0165540. doi: 10.1371/journal.pone.0165540

55. Lenfeldt N, Eriksson J, Åström B, Forsgren L, Mo SJ. Fractional anisotropy and mean diffusion as measures of dopaminergic function in parkinson's disease: challenging results. J Parkinsons Dis. (2017) 7:129-42. doi: 10.3233/JPD-161011

56. Taylor KI, Sambataro F, Boess F, Bertolino A, Dukart J. Progressive decline in gray and white matter integrity in de novo Parkinson's disease: an analysis of longitudinal Parkinson progression markers initiative diffusion tensor imaging data. Front Aging Neurosci. (2018) 10:318. doi: $10.3389 /$ fnagi.2018.00318

57. Pyatigorskaya N, Mongin M, Valabregue R, Yahia-Cherif L, Ewenczyk C, Poupon C, et al. Medulla oblongata damage and cardiac autonomic dysfunction in Parkinson disease. Neurology. (2016) 87:2540-5 doi: 10.1212/WNL.0000000000003426

58. Prange S, Metereau E, Maillet A, Lhommée E, Klinger H, Pelissier P, et al. Early limbic microstructural alterations in apathy and depression in de novo Parkinson's disease. Mov Disord. (2019) 34:1644-54. doi: 10.1002/mds.27793

59. Unger MM, Belke M, Menzler K, Heverhagen JT, Keil B, Stiasny-Kolster $\mathrm{K}$, et al. Diffusion tensor imaging in idiopathic REM sleep behavior disorder reveals microstructural changes in the brainstem, substantia nigra, olfactory region, and other brain regions. Sleep. (2010) 33:767-73. doi: $10.1093 /$ sleep/33.6.767

60. García-Lorenzo D, Longo-Dos Santos C, Ewenczyk C, Leu-Semenescu S, Gallea C, Quattrocchi G, et al. The coeruleus/subcoeruleus complex in rapid eye movement sleep behaviour disorders in Parkinson's disease. Brain. (2013) 136:2120-9. doi: 10.1093/brain/awt152

61. Pyatigorskaya N, Gaurav R, Arnaldi D, Leu-Semenescu S, Yahia-Cherif L, Valabregue R, et al. Magnetic resonance imaging biomarkers to assess substantia nigra damage in idiopathic rapid eye movement sleep behavior disorder. Sleep. (2017) 40. doi: 10.1093/sleep/zsx149

62. Youn J, Lee JM, Kwon H, Kim JS, Son TO, Cho JW. Alterations of mean diffusivity of pedunculopontine nucleus pathway in Parkinson's disease patients with freezing of gait. Park Relat Disord. (2015) 21:12-7. doi: 10.1016/j.parkreldis.2014.10.003

63. Heim B, Krismer F, Seppi K. Structural imaging in atypical parkinsonism. Int Rev Neurobiol. (2018)142:67-148. doi: 10.1016/bs.irn.2018.08.010

64. Seki M, Seppi K, Mueller C, Potrusil T, Goebel G, Reiter E, et al. Diagnostic potential of multimodal MRI markers in atypical parkinsonian disorders. $J$ Parkinsons Dis. (2019) 9:681-91. doi: 10.3233/JPD-181568

65. Talai AS, Sedlacik J, Boelmans K, Forkert ND. Widespread diffusion changes differentiate Parkinson's disease and progressive supranuclear palsy. NeuroImage Clin. (2018) 20:1037-43. doi: 10.1016/j.nicl.2018.09.028

66. Pasternak O, Sochen N, Gur Y, Intrator N, Assaf Y. Free water elimination and mapping from diffusion MRI. Magn Reson Med. (2009) 62:717-30. doi: $10.1002 / \mathrm{mrm} .22055$

67. Pasternak O, Westin C-F, Bouix S, Seidman LJ, Goldstein JM, Woo TUW, et al. Excessive extracellular volume reveals a neurodegenerative pattern in schizophrenia onset. J Neurosci. (2012) 32:17365-72. doi: 10.1523/JNEUROSCI.2904-12.2012

68. Ofori E, Pasternak O, Planetta PJ, Li H, Burciu RG, Snyder AF, et al Longitudinal changes in free-water within the substantia nigra of Parkinson's disease. Brain. (2015) 138:2322-31. doi: 10.1093/brain/awv136

69. Ofori E, Krismer F, Burciu RG, Pasternak O, McCracken JL, Lewis $\mathrm{MM}$, et al. Free water improves detection of changes in the substantia nigra in parkinsonism: a multisite study. Mov Disord. (2017) 32:1457-64. doi: $10.1002 / \mathrm{mds} .27100$

70. Arribarat G, Pasternak O, De Barros A, Galitzky M, Rascol O, Péran P. Substantia nigra locations of iron-content, free-water and mean diffusivity abnormalities in moderate stage Parkinson's disease. Parkinsonism Relat Disord. (2019) 65:146-152. doi: 10.1016/j.parkreldis.2019. 05.033

71. Burciu RG, Ofori E, Archer DB, Wu SS, Pasternak O, McFarland NR, et al. Progression marker of Parkinson's disease: a 4-year multi-site imaging study. Brain. (2017) 140:2183-92. doi: 10.1093/brain/awx146

72. Guttuso T, Bergsland N, Hagemeier J, Lichter DG, Pasternak O, Zivadinov R. Substantia nigra free water increases longitudinally in parkinson disease. $\mathrm{Am}$ J Neuroradiol. (2018) 39:479-84. doi: 10.3174/ajnr.A5545

73. Yang J, Archer DB, Burciu RG, Müller MLTM, Roy A, Ofori E, et al Multimodal dopaminergic and free-water imaging in Parkinson's disease. Park Relat Disord. (2019) 62:10-5. doi: 10.1016/j.parkreldis.2019.01.007

74. Planetta PJ, Ofori E, Pasternak O, Burciu RG, Shukla P, DeSimone JC, et al. Free-water imaging in Parkinson's disease and atypical parkinsonism. Brain. (2016) 139(Pt 2):495-508. doi: 10.1093/brain/awv361

75. Jelescu IO, Budde MD. Design and validation of diffusion MRI models of white matter. Front Phys. (2017) 5:61. doi: 10.3389/fphy.2017.00061

76. Wang JJ, Lin WY, Lu CS, Weng YH, Ng SH, Wang CH, et al. Parkinson disease: diagnostic utility of diffusion kurtosis imaging. Radiology. (2011) 261:210-7. doi: 10.1148/radiol.11102277

77. Zhang H, Schneider T, Wheeler-Kingshott CA, Alexander DC. NODDI: practical in vivo neurite orientation dispersion and density 
imaging of the human brain. Neuroimage. (2012) 61:1000-16. doi: 10.1016/j.neuroimage.2012.03.072

78. Kamagata K, Hatano T, Okuzumi A, Motoi Y, Abe O, Shimoji K, et al. Neurite orientation dispersion and density imaging in the substantia nigra in idiopathic Parkinson disease. Eur Radiol. (2016) 26:2567-77. doi: 10.1007/s00330-015-4066-8

79. Mitchell T, Archer DB, Chu WT, Coombes SA, Lai S, Wilkes BJ, et al. Neurite orientation dispersion and density imaging (NODDI) and freewater imaging in Parkinsonism. Hum Brain Mapp. (2019) 40:5094-107. doi: $10.1002 / \mathrm{hbm} .24760$

80. White NS, Leergaard TB, D'Arceuil H, Bjaalie JG, Dale AM. Probing tissue microstructure with restriction spectrum imaging: histological and theoretical validation. Hum Brain Mapp. (2013) 34:327-46. doi: 10.1002/hbm.21454

81. Hope TR, Selnes P, Rektorová I, Anderkova L, Nemcova-Elfmarkova N, BaláŽová Z, et al. Diffusion tensor and restriction spectrum imaging reflect different aspects of neurodegeneration in Parkinson's disease. PLOS ONE. (2019) 14:e0217922. doi: 10.1371/journal.pone.0217922

82. Lenglet C, Abosch A, Yacoub E, De Martino F, Sapiro G, Harel N. Comprehensive in vivo mapping of the human basal ganglia and thalamic connectome in individuals using 7T MRI. PLOS ONE. (2012) 7:e29153. doi: 10.1371/journal.pone.0029153

83. Plantinga BR, Roebroeck A, Kemper VG, Uludag K, Melse M, Mai J, et al. Ultra-high field MRI post mortem structural connectivity of the human subthalamic nucleus, Substantia nigra, and globus pallidus. Front Neuroanat. (2016) 10:66. doi: 10.3389/fnana.2016.00066

84. Abulaiti A, Kamagata K, Motoi Y, Hori M, Hattori N, Aoki S. Nigrostriatal degeneration in parkinson disease: evaluation by diffusion tensor tract-specific analysis. Open J Radiol. (2015) 05:199-204. doi: 10.4236/ojrad.2015.54027

85. Andica C, Kamagata K, Hatano T, Okuzumi A, Saito A, Nakazawa $\mathrm{M}$, et al. Neurite orientation dispersion and density imaging of the nigrostriatal pathway in Parkinson's disease: Retrograde degeneration observed by tract-profile analysis. Park Relat Disord. (2018) 51:55-60. doi: 10.1016/j.parkreldis.2018.02.046

86. Schneider TM, Deistung A, Biedermann U, Matthies C, Ernestus RI, Volkmann J, et al. Susceptibility sensitive magnetic resonance imaging displays pallidofugal and striatonigral fiber tracts. Oper Neurosurg. (2016) 12:330-8. doi: 10.1227/NEU.0000000000001256
87. Surova Y, Nilsson M, Lätt J, Lampinen B, Lindberg O, Hall S, et al. Disease-specific structural changes in thalamus and dentatorubrothalamic tract in progressive supranuclear palsy. Neuroradiology. (2015) 57:1079-91. doi: 10.1007/s00234-015-1563-z

88. Nilsson C, Markenroth Bloch K, Brockstedt S, Lätt J, Widner H, Larsson EM. Tracking the neurodegeneration of parkinsonian disorders - a pilot study. Neuroradiology. (2007) 49:111-9. doi: 10.1007/s00234-006-0165-1

89. Yang H, Wang X, Liao W, Zhou G, Li L, Ouyang L. Application of diffusion tensor imaging in multiple system atrophy: the involvement of pontine transverse and longitudinal fibers. Int J Neurosci. (2015) 125:18-24. doi: 10.3109/00207454.2014.896914

90. Ito K, Sasaki M, Ohtsuka C, Yokosawa S, Harada T, Uwano $\mathrm{I}$, et al. Differentiation among parkinsonisms using quantitative diffusion kurtosis imaging. Neuroreport. (2015) 26:267-72. doi: 10.1097/WNR.0000000000000341

91. Ito K, Ohtsuka C, Yoshioka K, Kameda H, Yokosawa S, Sato R, et al. Differential diagnosis of parkinsonism by a combined use of diffusion kurtosis imaging and quantitative susceptibility mapping. Neuroradiology. (2017) 59:759-69. doi: 10.1007/s00234-017-1870-7

92. Juttukonda MR, Franco G, Englot DJ, Lin YC, Petersen KJ, Trujillo P, et al. White matter differences between essential tremor and Parkinson disease. Neurology. (2019) 92:E30-9. doi: 10.1212/WNL.0000000000 006694

93. Yang J, Burciu RG, Vaillancourt DE. Longitudinal progression markers of parkinson's disease: current view on structural imaging. Curr Neurol Neurosci Rep. (2018) 18:1-11. doi: 10.1007/s11910-018-0894-7

Conflict of Interest: The authors declare that the research was conducted in the absence of any commercial or financial relationships that could be construed as a potential conflict of interest.

Copyright (c) 2020 Arribarat, De Barros and Péran. This is an open-access article distributed under the terms of the Creative Commons Attribution License (CC BY). The use, distribution or reproduction in other forums is permitted, provided the original author(s) and the copyright owner(s) are credited and that the original publication in this journal is cited, in accordance with accepted academic practice. No use, distribution or reproduction is permitted which does not comply with these terms. 